\title{
Elective Laparoscopic Sigmoidectomy with Preservation of the Superior Rectal Artery Prior to Endoscopic Decompression in Sigmoid Volvulus
}

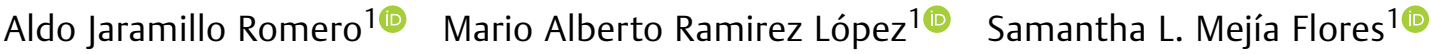 \\ Andrés Niño Carrasco ${ }^{10}$ \\ ${ }^{1}$ Department of Colorectal Surgery, Hospital Central Militar, Ciudad \\ Address for correspondence Aldo Jaramillo Romero, MD, \\ de México, Mexico \\ Department of Colorectal Surgery, Hospital Central Militar, Ciudad de \\ México 11200, CDMX, Mexico (e-mail: aldojr.26@hotmail.com). \\ J Coloproctol 2021;41(4):447-450.
}

\begin{abstract}
Sigmoid volvulus is a frequent cause of intestinal obstruction. Its management has evolved with the use of laparoscopic surgery, achieving an elective sigmoid resection with anastomosis after a flexible endoscopic detorsion. A female patient was admitted to the emergency room with abdominal pain, distention, and constipation. The abdominal computed tomography showed a whirled sigmoid mesentery in addition to dilated sigmoid loops, and coffee bean sign. The patient successfully underwent a flexible endoscopic detorsion and was scheduled for elective sigmoid colectomy with rectal superior artery preservation and primary anastomosis. During the sigmoid

Keywords

- intestinal volvulus

- sigmoid

- laparoscopy resection, the superior rectal artery preservation is related to a better prognostic, with less bleeding, anastomotic leakage, and hospital stay. Currently, there are few reports of the laparoscopic preservation of the superior rectal artery in patients with sigmoid volvulus.
\end{abstract}

\section{Introduction}

Volvulus is a condition in which the colon wraps around itself and its own mesentery, causing a closed loop obstruction. It accounts for up to between 50 and $80 \%$ of intestinal obstructions. ${ }^{1,2}$ The most common sites for volvulus are the sigmoid colon and the cecum. ${ }^{3-6}$

A flexible endoscopic detorsion can eliminate the volvulus by going forward through the compromised segment of the colon, restoring the passage of luminal content, and ensuring perfusion of the sigmoid colon. ${ }^{7}$ Flexible endoscopic detorsion is successful in up to between 75 and $90 \%$ of hemody- namically stable patients in absence of ischemic lesions, necrosis, or colon perforation. ${ }^{7}$

After the endoscopic detorsion, it is feasible to perform an elective sigmoidectomy with colorectal anastomosis instead of a Hartmann procedure in emergency surgery, achieving lower rates of postoperative complications, without observing a direct impact on mortality. ${ }^{8}$

The role of laparoscopic surgery in colorectal procedures continues growing, but there are only a few references in the published literature about the laparoscopic management of sigmoid volvulus. Various articles have established that the laparoscopic approach is an adequate alternative in selected received

November 11, 2020 accepted after revision June 15, 2021
DOI https://doi.org/

$10.1055 / \mathrm{s}-0041-1739315$. ISSN 2237-9363.

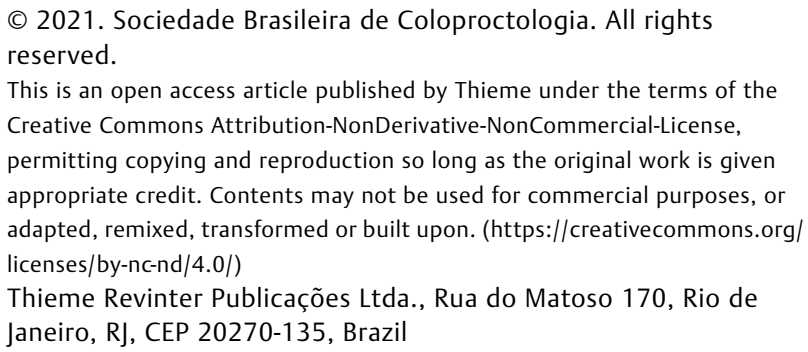




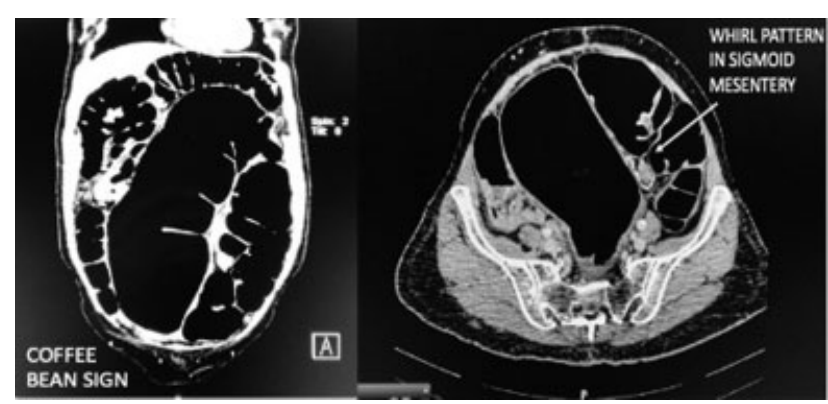

Fig. 1 Abdominal computed tomography showing the characteristic images of volvulus: coffee bean sign (left) and whirl pattern in the sigmoid mesentery (right).

cases by surgeons competent with this technique. ${ }^{9,10}$ Recent studies have determined better outcomes when preserving the superior rectal artery (SRA) in patients with sigmoid resection, which is a more complex procedure when performed through laparoscopic surgery.

We present the surgical management of a patient with sigmoid volvulus performing an endoscopic colonic decompression and elective laparoscopic sigmoidectomy with colorectal anastomosis, preserving the SRA.

\section{Clinical Case}

A 64-year-old female patient presented to the emergency room with abdominal pain, distension, and constipation of 24 hours of evolution. She had a personal history of type 2 diabetes mellitus of 30 years of evolution and hysterectomy in 2016. Physical examination revealed a distended abdomen, with pain at palpation over the left hemiabdomen, tympanic at percussion with no evidence of peritoneal irritation. Blood tests showed no systemic inflammatory response, leucocytes $7,200 \mu \mathrm{L}$, C-reactive protein $1 \mathrm{mg} / \mathrm{L}$, and lactate $1.5 \mathrm{mmol} / \mathrm{L}$. The abdominal computed tomography (CT) showed dilated large bowel loops, the classic image of coffee bean sign (-Fig. 1A), and a whirl pattern in the sigmoid mesentery (-Fig. 1B). After confirmation of the diagnosis and initial management with fluid and antibiotics, a colonoscopy to decompress the volvulus was performed. During the procedure, an increased compliance and elasticity was observed, as well as an altered haustra distribution and vascular pattern, increased intestinal caliber, and generalized edema, with no evidence of ischemia or perforation. The colonoscope was passed toward the descending and transverse colon and up to the hepatic flexure, with subsequent successful colonic decompression (-Fig. 2). Forty-eight hours after the colonoscopy, a laparoscopic sigmoidectomy was performed. Under direct vision, redundant sigmoid was observed. The sacral promontory was identified to start the peritoneum dissection $2 \mathrm{~cm}$ above it, recognizing the inferior mesenteric artery and the SRA, delimiting its path to its origin for subsequent preservation (-Fig. 3). After we finished the dissection of the toldt fascia, we began the resection of the mesorectum. A linear laparoscopic stapler was used for its resection. A wide incision was made in the umbilical region for placement of a wound protector retractor (Alexis ${ }^{\circledR}$ C8301 Applied Medical, USA) and exteriorization of the surgical piece with its resection on viable tissue (-Fig. 4). End-to-end colorectal anastomosis was performed with a circular stapler (-Fig. 5). At the end, a pneumatic test and flexible intraoperative rectosigmoidoscopy were performed, identifying a complete anastomosis, with no evidence of leakage or bleeding. The surgical piece was sent to pathology, which reported mild chronic colitis, with no data of malignancy in any segment, both discoid specimens were intact and uniform. Surgery was terminated without complications, with minimal bleeding of $50 \mathrm{cc}$ at a registered time of 150 minutes. The patient presented adequate postoperative evolution, starting to eat on the $1^{\text {st }}$ postoperative day, with adequate tolerance, gas channeling, and

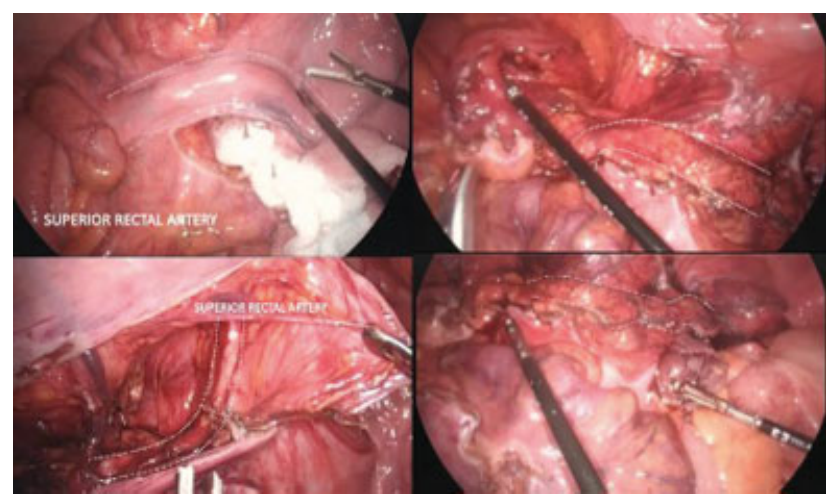

Fig. 3 Identification of the superior rectal artery after dissection of peritoneum, delimitating the path of the superior rectal artery toward its origin.

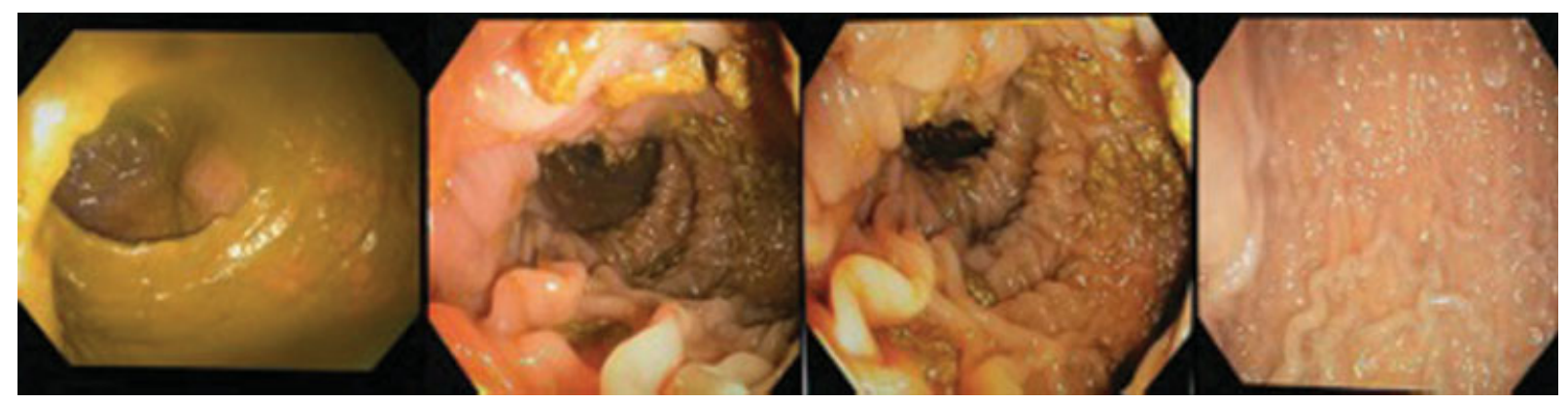

Fig. 2 Colonic decompression by flexible rectosigmoidoscopy; we observe altered haustra distribution and vascular pattern, without signs of ischemic zones. 


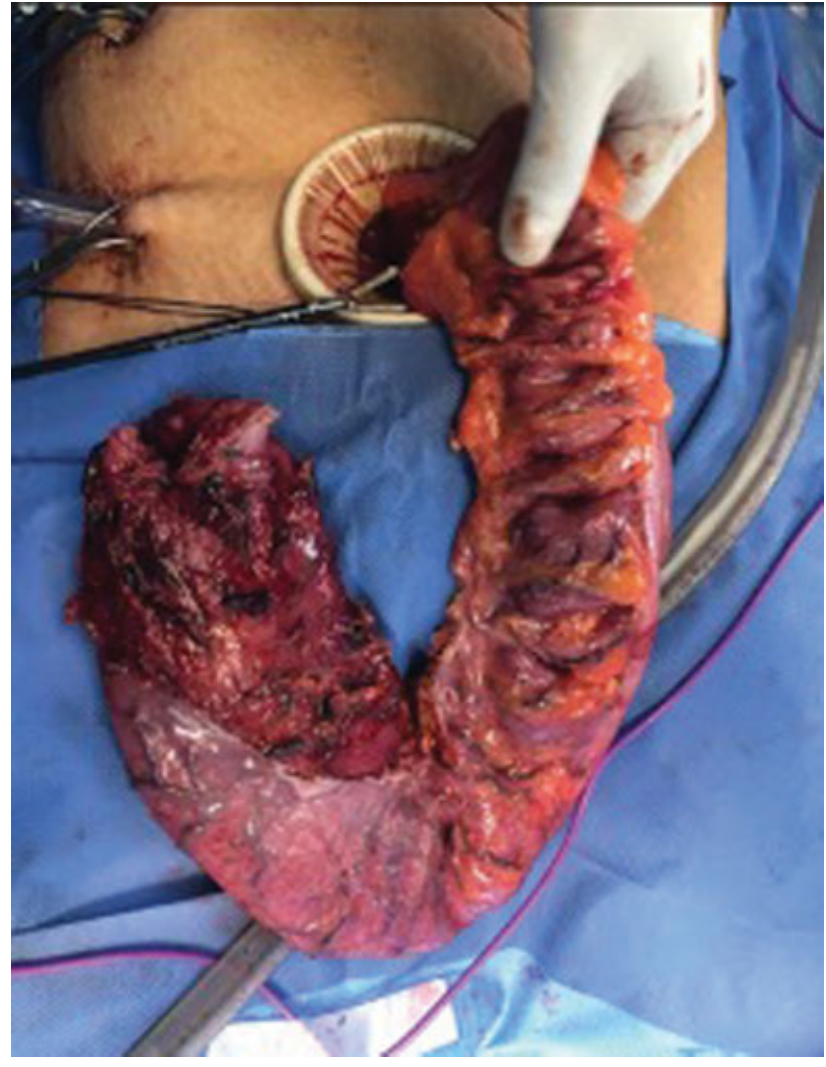

Fig. 4 Exteriorization of the surgical piece. Redundant sigmoid is observed.

consistent evacuations present, and was discharged on the $4^{\text {th }}$ postoperative day.

\section{Discussion}

Elective laparoscopic surgery after adequate endoscopic decompression for sigmoid volvulus has made this type of procedure feasible and safe in patients with hemodynamic stability, especially when performed by a trained team with surgical experience in laparoscopic procedures. After endoscopic decompression, the time for the colectomy remains a matter of discussion. Most studies suggest operating on between the $3^{\text {rd }}$ and $5^{\text {th }}$ day after the decompression. There are few publications regarding the benefits of early versus late colectomy. Some benefits of making the colectomy earlier include decreased deconditioning, malnutrition, and length of stay. On the other hand, a late colectomy is related to decreased bowel wall edema and, possibly, with an increased likelihood of an anastomosis. ${ }^{11}$

Preservation of the SRA is associated with a favorable outcome in patients undergoing laparoscopic sigmoid resection for diverticular disease. Maximilian Sohn et al. ${ }^{12}$ reported lower data of anastomotic leakage in patients with preservation of the SRA compared with patients in whom it has been resected. ( 1.9 versus $7 \% ; p=0.04$ ). Borchert et al. $^{13}$ conducted a study in which they compared patients with preservation of the SRA with patients who had it resected, observing that patients with preservation of the SRA had a lower incidence of anastomotic leakage (1

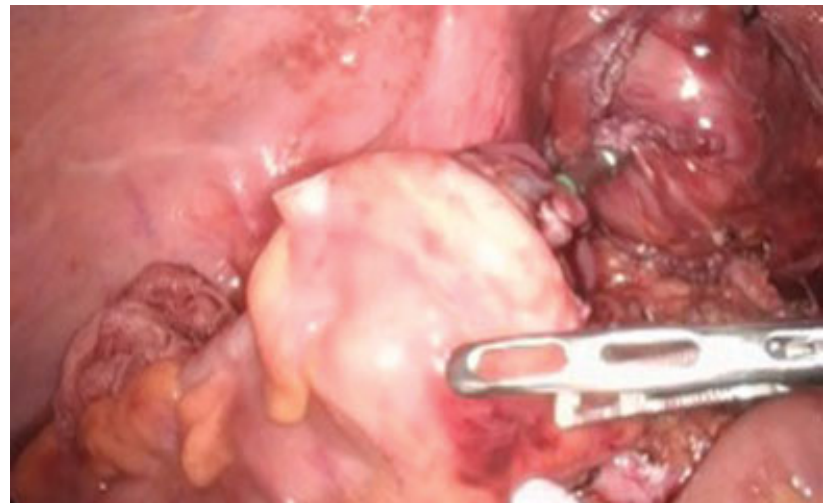

Fig. 5 Colorectal anastomosis with circular stapler.

versus 8 patients; $p=0.038$ ). The incidence of intraoperative bleeding, wound dehiscence, and days of hospital stay was higher in patients in whom the SRA was resected ( $p<0.03$; $p<0.04 ; p=0.05)$. Obesity was an independent risk factor for anastomotic leakage in these patients $(p<0.04)$.

There are few articles in the literature with reference to the preservation of the SRA in patients with sigmoidectomy secondary to sigmoid volvulus. The published literature refers only to patients with diverticular disease in this type of procedure. The report of the present case and the published literature on the preservation of the SRA have shown this to be a feasible procedure, without increasing the operative time and with a reduction in the risks of postoperative complications in patients selected for laparoscopic sigmoid resection.

\section{Conflict of Interests}

The authors have no conflict of interests to declare.

\section{References}

1 Ballantyne GH, Brandner MD, Beart RW Jr, Ilstrup DM. Volvulus of the colon. Incidence and mortality. Ann Surg 1985;202(01):83-92

2 Friedman JD, Odland MD, Bubrick MP. Experience with colonic volvulus. Dis Colon Rectum 1989;32(05):409-416

3 Asbun HJ, Castellanos H, Balderrama B, et al. Sigmoid volvulus in the high altitude of the Andes. Review of 230 cases. Dis Colon Rectum 1992;35(04):350-353

4 Gama AH, Haddad J, Simonsen O, et al. Volvulus of the sigmoid colon in Brazil: a report of 230 cases. Dis Colon Rectum 1976;19 (04):314-320

5 Halabi WJ, Jafari MD, Kang CY, et al. Colonic volvulus in the United States: trends, outcomes, and predictors of mortality. Ann Surg 2014;259(02):293-301

6 Shepherd JJ. The epidemiology and clinical presentation of sigmoid volvulus. Br J Surg 1969;56(05):353-359

7 Atamanalp SS. Treatment of sigmoid volvulus: a single-center experience of 952 patients over 46.5 years. Tech Coloproctol 2013;17(05):561-569

8 Oren D, Atamanalp SS, Aydinli B, et al. An algorithm for the management of sigmoid colon volvulus and the safety of primary resection: experience with 827 cases. Dis Colon Rectum 2007;50 (04):489-497

9 Liang JT, Lai HS, Lee PH. Elective laparoscopically assisted sigmoidectomy for the sigmoid volvulus. Surg Endosc 2006;20(11): $1772-1773$ 
450 Elective Laparoscopic Sigmoidectomy with Preservation of the Superior Rectal Artery Jaramillo et al.

10 Sigmoid Volvulus with Widespread Bowel Ischemia after Endoscopic Reduction Successfully Treated with Elective Laparoscopic Surger; koji yasuda et al. Case Rep Gastroenterol 2020; 14:286-290; S.Karger AG, Basel

11 Garfinkle R, Morin N, Ghitulescu G, Vasilevsky CA, Boutros M. From Endoscopic Detorsion to Sigmoid Colectomy-The Art of Managing Patients with Sigmoid Volvulus: A Survey of the Members of the American Society of Colon and Rectal Surgeons. Am Surg 2018;84(09):1518-1525
12 Maximilian Sohnet al.Preservation of the superior rectal artery: influence of surgical technique on anastomotic healing and postoperative morbidity in laparoscopic sigmoidectomy for diverticular disease. Int J Colorectal Dis Springer-Verlag Berlin Heidelberg; 2017

13 Borchert DH, Schachtebeck M, Schoepe J, Federlein M, Bunse J, Gellert K, Burghdart J. Observational study on preservation of the superior rectal artery in sigmoid resection for diverticular disease. International Journal of Surgery 21 2015:45-50 